\title{
Blood Glucose Test Strip Quantity Limits Across Canada
}

Research has suggested that frequent monitoring of blood glucose for patients who do not take insulin to manage their diabetes is not considered clinically beneficial and may even lead to decreased quality of life and well-being.

Provincial governments across Canada have implemented blood glucose test strip (BGTS) quantity limits to encourage proper testing practices for optimal patient outcomes. See below a provincial list of implementation details as of June 2017.

\begin{tabular}{|c|c|c|c|}
\hline $\begin{array}{l}\text { Provincel } \\
\text { Payer }\end{array}$ & Implementation Date & Details & Link \\
\hline Alberta & July 1, 2012 & $\begin{array}{l}\text { Insulin users only - Coverage to a maximum of } \$ 600 \text { per person each } \\
\text { benefit year for eligible diabetic supplies. }\end{array}$ & Government of Alberta \\
\hline British Columbia* & January 1, 2015 & $\begin{array}{l}\text { Insulin users - 3,000 strips per year } \\
\text { High Hypoglycemic risk medication users - } 400 \text { strips per year } \\
\text { Low Hypoglycemic risk medication users - } 200 \text { strips per year } \\
\text { No medications - } 200 \text { strips per year }\end{array}$ & $\underline{\text { Government of British }}$ \\
\hline Manitoba* & June 15, 2017 & $\begin{array}{l}\text { Insulin users - 3,650 strips per year } \\
\text { High Hypoglycemic risk medication users - } 400 \text { strips per year } \\
\text { Low Hypoglycemic risk medication users - } 200 \text { strips per year } \\
\text { No medications - } 200 \text { strips per year }\end{array}$ & Government of Manitoba \\
\hline New Brunswick* & April 1, 2013 & $\begin{array}{l}\text { Insulin users or pregnant - Individualized (no limit) } \\
\text { Type } 2 \text { diabetes oral medication users - } 100 \text { strips per year } \\
\text { Newly diagnosed type } 2 \text { diabetes no medications - } 50 \text { strips per year }\end{array}$ & $\begin{array}{l}\text { Government of New } \\
\text { Brunswick }\end{array}$ \\
\hline $\begin{array}{l}\text { Newfoundland } \\
\text { and Labrador }\end{array}$ & July 1, 2016 & $\begin{array}{l}\text { Insulin users - 2,500 strips per year } \\
\text { High Hypoglycemic risk medication users - } 700 \text { strips per year } \\
\text { Low Hypoglycemic risk medication users }-100 \text { strips per year } \\
\text { No medications - } 50 \text { strips per year }\end{array}$ & $\begin{array}{l}\text { Government of } \\
\text { Newfoundland and } \\
\text { Labrador }\end{array}$ \\
\hline Nova Scotia & No limits in place. & Limit to the amount prescribed. & \\
\hline Ontario* & August 1, 2013 & $\begin{array}{l}\text { Insulin users - 3,000 strips per year } \\
\text { High Hypoglycemic risk medication users }-400 \text { strips per year } \\
\text { Low Hypoglycemic risk medication users }-200 \text { strips per year } \\
\text { No medications - } 200 \text { strips per year }\end{array}$ & $\begin{array}{l}\text { Ontario Ministry of } \\
\underline{\text { Health and Long-Term }} \\
\underline{\text { Care }}\end{array}$ \\
\hline $\begin{array}{l}\text { Prince Edward } \\
\text { Island }\end{array}$ & November 13, 2008 & $\begin{array}{l}\text { Insulin users only - } 100 \text { strips every } 30 \text { days ( } 1,200 \text { Annual Limit) } \\
\text { if registered with the Diabetes Drug Program and/or Catastrophic } \\
\text { Drug Program and have taken insulin within the past } 5 \text { months. } \\
\text { The patient is responsible for an } \$ 11.00 \text { co-pay per box of strips. }\end{array}$ & $\begin{array}{l}\text { Government of Prince } \\
\text { Edward Island }\end{array}$ \\
\hline Québec & May 3, 2017 & $\begin{array}{l}\text { Insulin users - 3,000 strips per year } \\
\text { High Hypoglycemic risk medication users - } 400 \text { strips per year } \\
\text { Low Hypoglycemic risk medication users - } 200 \text { strips per year } \\
\text { No medications - } 200 \text { strips per year }\end{array}$ & Government of Québec \\
\hline Saskatchewan* & October 15, 2015 & $\begin{array}{l}\text { Insulin users - 3,650 strips per year } \\
\text { High Hypoglycemic risk medication users }-400 \text { strips per year } \\
\text { Low Hypoglycemic risk medication users - } 200 \text { strips per year } \\
\text { No medications - } 200 \text { strips per year }\end{array}$ & $\frac{\text { Government of }}{\text { Saskatchewan }}$ \\
\hline Yukon & Not available. & Insulin users only - 10 strips per day & \\
\hline $\begin{array}{l}\text { Non-Insured } \\
\text { Health Benefits } \\
\text { (NIHB) }\end{array}$ & $\begin{array}{l}\text { November 4, } 2013 \text { (Atlantic) } \\
\text { January 6, } 2014 \text { (ON) } \\
\text { March 3, } 2014 \text { (Northern) } \\
\text { April 7, } 2014 \text { (AB) } \\
\text { June 2, } 2014 \text { (SK) } \\
\text { July 7, } 2014 \text { (MB) } \\
\text { September 2, } 2014 \text { (QC) } \\
\text { January 15, } 2015 \text { (BC) }\end{array}$ & $\begin{array}{l}\text { Insulin users - } 500 \text { strips per } 100 \text { days } \\
\text { High Hypoglycemic risk medication users }-400 \text { strips per year } \\
\text { Low Hypoglycemic risk medication users - } 200 \text { strips per year } \\
\text { No medications - } 200 \text { strips per year }\end{array}$ & NIHB Program \\
\hline
\end{tabular}

*Province will cover additional 100 strips per year permitted in exceptional circumstances. In BC, broader overrides are permitted upon request.

\section{Last updated June 2017}

Compiled by the Ontario Drug Policy Research Network for informational purposes only.

Amendments or errors can be sent to: info@odprn.ca

For more information: http://odprn.ca/research/core-themes/self-monitoring-of-blood-glucose/ 